\title{
Perioperative factors associated with pressure ulcer development after major surgery
}

\author{
Jeong Min Kim, Hyunjeong Lee, Taehoon $\mathrm{Ha}$, and Sungwon $\mathrm{Na}$ \\ Department of Anesthesiology and Pain Medicine, Anesthesia and Pain Research Institute, Yonsei University \\ College of Medicine, Seoul, Korea
}

Background: Postoperative pressure ulcers are important indicators of perioperative care quality, and are serious and expensive complications during critical care. This study aimed to identify perioperative risk factors for postoperative pressure ulcers.

Methods: This retrospective case-control study evaluated 2,498 patients who underwent major surgery. Forty-three patients developed postoperative pressure ulcers and were matched to 86 control patients based on age, sex, surgery, and comorbidities.

Results: The pressure ulcer group had lower baseline hemoglobin and albumin levels, compared to the control group. The pressure ulcer group also had higher values for lactate levels, blood loss, and number of packed red blood cell ( $p$ RBC) units. Univariate analysis revealed that pressure ulcer development was associated with preoperative hemoglobin levels, albumin levels, lactate levels, intraoperative blood loss, number of $p \mathrm{RBC}$ units, Acute Physiologic and Chronic Health Evaluation II score, Braden scale score, postoperative ventilator care, and patient restraint. In the multiple logistic regression analysis, only preoperative low albumin levels (odds ratio [OR]: $0.21,95 \% \mathrm{CI}: 0.05-0.82 ; \mathrm{P}<0.05$ ) and high lactate levels (OR: 1.70, 95\% CI: 1.07-2.71; P < 0.05) were independently associated with pressure ulcer development. A receiver operating characteristic curve was used to assess the predictive power of the logistic regression model, and the area under the curve was 0.88 (95\% CI: 0.79-0.97; P $<0.001$ ).

Conclusions: The present study revealed that preoperative low albumin levels and high lactate levels were significantly associated with pressure ulcer development after surgery.

Keywords: Albumin; Lactate; Perioperative risk factors; Pressure ulcer.

Corresponding author: Sungwon Na, M.D., Ph.D.

Department of Anesthesiology and Pain Medicine, Anesthesia and Pain Research Institute, Yonsei University College of Medicine, 50, Yonsei-ro, Seodaemun-gu, Seoul 03722, Korea

Tel: 82-2-2227-4237, Fax: 82-2-2227-7897

Email: nswksj@yuhs.ac

ORCID: https://orcid.org/0000-0002-1170-8042

Received: December 1, 2016.

Revised: April 8, 2017 (1st); May 22, 2017 (2nd).

Accepted: May 23, 2017.

Korean J Anesthesiol 2018 February 71(1): 48-56 https://doi.org/10.4097/kjae.2018.71.1.48

\section{Introduction}

Postoperative pressure ulcers are important indicators of perioperative care quality and are significant and expensive complications during critical care that can result in unexpected morbidity. A report from the Centers for Medicare and Medicaid Services revealed that Medicare pays approximately $\$ 146$ million per year to cover treatment for six hospital-acquired conditions, including severe pressure ulcers, and that pressure ulcers had the second highest cost per episode [1]. Therefore, the prevention of pressure ulcers can help reduce healthcare costs and improve patient outcomes.

Anesthesiologists may not consider the risk of postoperative

(c) This is an open-access article distributed under the terms of the Creative Commons Attribution Non-Commercial License (http://creativecommons.org/ licenses/by-nc/4.0/), which permits unrestricted non-commercial use, distribution, and reproduction in any medium, provided the original work is properly cited. 
pressure ulcers, as only patient positioning has been considered the primary responsibility of anesthesiologists [2]. However, postoperative pressure ulcer development is associated with many other variables, such as the length of surgery, and anesthesiologists' limited inability to control these variables may have prevented them from playing a more active role in pressure ulcer prevention. Furthermore, there is no ideal method for identifying patients who are at risk of developing postoperative pressure ulcers. The Braden scale has been used for surgical patients, although it has low sensitivity and modest specificity for predicting pressure ulcers [3].

O'Brien et al. [4] have recently investigated intraoperative risk factors for postoperative pressure ulcers and found that only the intraoperative use of blood products was independently associated with pressure ulcer development. No significant associations were observed for operation length, hypotension, or vasopressor use. These findings highlight the possibility that pressure ulcer development is not related to distinct intraoperative factors, although that study did not consider the association of the patients' pre-existing conditions (e.g., nutritional status) with pressure ulcer occurrence. In addition, there is a validated scoring system that is commonly used in the critical care setting to identify patients with a high risk of developing pressure ulcers [5], although the score may not be updated to reflect the patient's altered mobility after surgery [6]. Therefore, the present study aimed to identify perioperative factors that could identify patients who were at risk of developing postoperative pressure ulcers and to develop a model for predicting pressure ulcer development after surgery.

\section{Materials and Methods}

This retrospective case-control study evaluated data from 129 patients who underwent major surgery between January 2012 and December 2013 at the Severance Hospital of the Yonsei University Health System (Seoul, Korea). Institutional Review Board approval was obtained (4-2014-0323) and this study was registered at www.ClinicalTrials.gov (NCT02279355). The exclusion criteria were pediatric cases and cases with preoperative pressure ulcers. The patients' baseline data included demographic characteristics, physiological parameters, comorbidities, functional status, and nutritional status. Each case was paired with two controls that were matched for age ( \pm 5 years), sex, surgery, and comorbidities within the same period.

The potentially eligible population included 2,498 patients who were discharged from the surgical intensive care unit (ICU) after surgery. New-onset postoperative pressure ulcers (within one month after surgery) were identified based on nursing notes in the patients' electronic medical records. Based on the pressure ulcer staging guidelines of the National Pressure Ulcer Advisory
Panel [7], 43 patients were found to have developed pressure ulcers of stage 2 or higher (stage 2: 28 patients, stage 3: 12 patients, deep tissue injury: 1 patient, unstageable ulcers: 2 patients). In this staging system, the degree of tissue damage increases at higher stages. Stage 1 involves intact skin with a localized area of non-blanchable erythema, which may appear different in darkly pigmented skin and is usually temporary and reversible. Because of their immobilization, patients commonly experience a stage 1 pressure ulcer at weight-bearing sites immediately after surgery. Therefore, the initial management of stage 1 pressure ulcers only involves a protective dressing with observation, and stage 1 pressure ulcers are not routinely treated by a wound care nurse. Stage 2 involves partial-thickness skin loss with exposed dermis in a red or irritated area and can involve a blister or open sore. Stage 3 involves full-thickness skin loss with adipose tissue that is visible in the ulcer, and granulation tissue and epibole are often present. Stage 4 involves full-thickness skin and tissue loss with exposed or directly palpable fascia, muscle, tendon, ligament, cartilage, or bone. Unstageable pressure ulcers involve full-thickness skin and tissue loss that is obscured by slough or eschar, which prevents direct visualization of the extent of tissue damage. If the slough or eschar is removed, a stage 3 or 4 pressure injury will be revealed. Deep tissue pressure injury involves persistent non-blanchable deep red, maroon, or purple discoloration.

The preoperative data included comorbidities, age, sex, body mass index (BMI), corticosteroid use, diabetes, neurological disease, serum albumin levels, hemoglobin levels, creatinine levels, prothrombin time, hemoglobin levels, total bilirubin levels, and lactate levels. The intraoperative data included duration of anesthesia, intraoperative patient position, anesthesia type (general or regional anesthesia), hypotension (a 40\% relative decrease in mean arterial pressure compared with baseline mean arterial pressure), vasopressor use, volume of blood loss, number of packed red blood cell ( $p$ RBC) units transfused, and intraoperative urine output. The postoperative data during the first week included the length of ICU stay, length of hospital stay, Acute Physiologic and Chronic Health Evaluation (APACHE II) score, Braden scale score, use of restraints, total parenteral nutrition, ventilator care, use of vasopressors, in-hospital mortality, and laboratory data, which included prothrombin time and levels of serum albumin, hemoglobin, creatinine, total bilirubin, and lactate. The Braden scale is a tool for predicting the risk of pressure ulcers, which was developed in 1987 by Braden and Bergstrom [8]. The Braden scale is based on six criteria: sensory perception, moisture, activity, mobility, dietary habits, and friction and shear. A nurse performed bedside assessments immediately after the patient arrived at the ICU. Patients who required postoperative ventilator care received sedatives and analgesics to maintain light sedation (Richmond agitation sedation scale: -2 to 0 ) 
which helped synchronize the patient's spontaneous breathing with the mechanical ventilation.

All statistical analyses were performed using R software (version 3.0.1; The R Foundation for Statistical Computing, Vienna, Austria). Results were considered statistically significant at a $\mathrm{P}$ value of $<0.05$. Categorical variables were evaluated using two-tailed Fisher exact tests for $2 \times 2$ tables and the likelihood ratio chi-square test was used for larger tables. Continuous variables were inspected visually and tested for normality using the Kolmogorov-Smirnov test. Normally distributed data were evaluated using a two-sample test, and non-normally distributed data were evaluated using the Mann-Whitney $U$ test. Survival rates were estimated using the Kaplan-Meier method. Risk factors that were significantly associated with postoperative pressure ulcer development were integrated into a logistic regression model. The predictive power of the model was evaluated using a receiver operating characteristic (ROC) curve and the area under the curve (AUC). The nomogram's discrimination and calibration were tested as previously reported [9], and the bootstrap method (200 repetitions) was used to obtain relatively unbiased estimates of the model's performance.

Discrimination (i.e., whether the relative rankings of individual predictors of postoperative pressure ulcers were in the correct order) was quantified using the AUC value and its 95\% CI. We also performed graphic calibrations of the relationship between the observed outcome and expected probabilities. The clinical significance of the calibration represents the accuracies of individual predictors of postoperative pressure ulcers.

\section{Results}

Among the 2,498 assessed patients, 43 patients developed postoperative stage 2 or higher pressure ulcers (Fig. 1). Each case and its two matched controls had identical values for the matched factors (Fig. 1). The patients' baseline characteristics are summarized in Table 1. The two groups had similar baseline values for diabetes, steroid use, BMI, and American Society of Anesthesiologists physical status classification score. Compared with the control group, the pressure ulcer group had lower baseline values for hemoglobin and albumin levels $(\mathrm{P}=0.014)$ and higher baseline lactate levels $(\mathrm{P}=0.001)$. Both groups had similar total anesthesia times $(\mathrm{P}=0.575)$. The pressure ulcer group had a higher volume of blood loss and more transfused $p \mathrm{RBC}$ units, compared with the control group $(P=0.002)$. Among the postoperative variables, the pressure ulcer group had higher APACHE scores $(P=0.003)$, higher Braden scale scores at admission $(\mathrm{P}<0.001)$, longer ICU stays $(\mathrm{P}<0.001)$, and higher likelihoods of requiring mechanical ventilation $(\mathrm{P}<0.001)$, physical restraint $(\mathrm{P}<0.001)$, and mortality $(\mathrm{P}<0.001)$. There were no intergroup differences in intraoperative positioning. The Kaplan-Meier survival estimates revealed that, compared with the control group, the pressure ulcer group had significant-

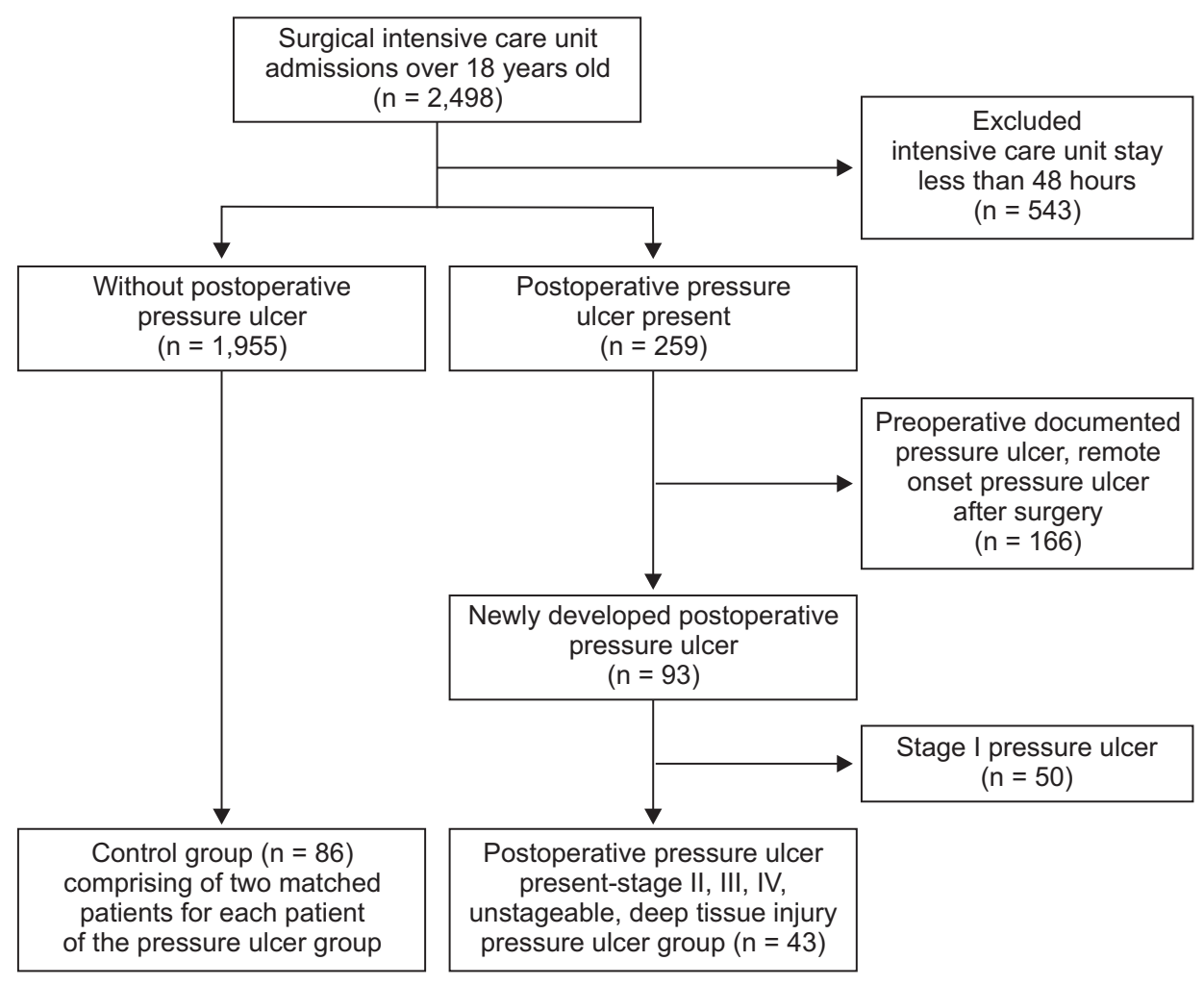

Fig. 1. Patient flow chart. 
Table 1. Perioperative Characteristics of the Patients

\begin{tabular}{|c|c|c|c|}
\hline & No Pressure ulcer $(\mathrm{n}=86)$ & Pressure ulcer $(\mathrm{n}=43)$ & $P$ value \\
\hline \multicolumn{4}{|l|}{ Preoperative characteristics } \\
\hline Age (yr) & $61.8 \pm 10.8$ & $61.7 \pm 10.7$ & 0.592 \\
\hline Male sex, $\mathrm{n}(\%)$ & $69(80 \%)$ & $35(84 \%)$ & 0.811 \\
\hline Body mass index $\left(\mathrm{kg} / \mathrm{m}^{2}\right)$ & $22.1 \pm 4.0$ & $23.3 \pm 4.3$ & 0.122 \\
\hline ASA class (1-3 vs. $4-5)$ & 60 vs. 26 & 24 vs. 19 & 0.123 \\
\hline Charlson comorbidity score & $63 \pm 11$ & $61 \pm 11$ & 0.175 \\
\hline Hemoglobin (g/dl) & $12.5 \pm 2.1$ & $11.6 \pm 1.9$ & 0.014 \\
\hline $\operatorname{Albumin}(\mathrm{g} / \mathrm{dl})$ & $3.7 \pm 0.6$ & $3.1 \pm 0.8$ & $<0.001$ \\
\hline Lactate $(\mathrm{mmol} / \mathrm{L})$ & $1.3 \pm 0.9$ & $3.2 \pm 2.9$ & 0.001 \\
\hline \multicolumn{4}{|l|}{ Intraoperative characteristics } \\
\hline Solid organ transplantation (liver \& lung) & $26(30 \%)$ & $13(30 \%)$ & \\
\hline Open abdominal surgery & $22(26 \%)$ & $11(26 \%)$ & \\
\hline Thoracic surgery & $18(21 \%)$ & $9(21 \%)$ & \\
\hline Orthopedic surgery & $6(7 \%)$ & $3(7 \%)$ & \\
\hline Head and neck surgery & $10(12 \%)$ & $5(12 \%)$ & \\
\hline Urological surgery & $2(2 \%)$ & $1(2 \%)$ & \\
\hline Laparoscopic abdominal surgery & $2(2 \%)$ & $1(2 \%)$ & \\
\hline Anesthesia time (min) & $460(247-660)$ & $310(215-670)$ & 0.575 \\
\hline Blood loss (ml) & $525(200-1,662)$ & $900(250-2,300)$ & 0.054 \\
\hline Packed RBC transfusion (units) & $0(0-2)$ & $1(0-5)$ & 0.002 \\
\hline Intraoperative minimum $\mathrm{pH}$ & $7.3 \pm 0.1$ & $7.3 \pm 0.1$ & 0.058 \\
\hline Hypotensive event (mean blood pressure $<40 \%$ of baseline), $\mathrm{n}(\%)$ & $37(43 \%)$ & $24(56 \%)$ & 0.193 \\
\hline Intraoperative vasopressor, $\mathrm{n}(\%)$ & $42(49 \%)$ & $26(61 \%)$ & 0.263 \\
\hline Minimum body temperature $\left({ }^{\circ} \mathrm{C}\right)$ & $35.6 \pm 0.9$ & $35.4 \pm 0.9$ & 0.328 \\
\hline Intraoperative patient position & & & 0.049 \\
\hline Supine & $57(66 \%)$ & $36(84 \%)$ & \\
\hline Prone & $4(5 \%)$ & $0(0 \%)$ & \\
\hline Lateral & $22(26 \%)$ & $7(16 \%)$ & \\
\hline Lithotomy & $3(4 \%)$ & $0(0 \%)$ & \\
\hline \multicolumn{4}{|l|}{ Postoperative characteristics } \\
\hline APACHE II score & $16.3 \pm 7.0$ & $20.1 \pm 6.2$ & 0.003 \\
\hline Braden scale & $14.4 \pm 2.8$ & $12.5 \pm 2.7$ & $<0.001$ \\
\hline $\mathrm{pH}$ & $7.37 \pm 0.07$ & $7.35 \pm 0.10$ & 0.197 \\
\hline Hemoglobin (g/dl) & $11.5 \pm 2.1$ & $10.2 \pm 2.3$ & 0.004 \\
\hline Lactate $(\mathrm{mmol} / \mathrm{L})$ & $3.0 \pm 2.6$ & $4.5 \pm 3.2$ & 0.017 \\
\hline Postoperative ICU stay (days) & $3(2-7)$ & $9(5-25)$ & $<0.001$ \\
\hline Postoperative hospital stay (days) & $21(13-35)$ & $28(14-54)$ & 0.471 \\
\hline Mortality, n (\%) & $8(9 \%)$ & $17(40 \%)$ & $<0.001$ \\
\hline Ventilator care, n (\%) & $45(52 \%)$ & $38(88 \%)$ & $<0.001$ \\
\hline Restraint, $\mathrm{n}(\%)$ & $45(52 \%)$ & $39(91 \%)$ & $<0.001$ \\
\hline Total parenteral nutrition, $\mathrm{n}(\%)$ & $40(47 \%)$ & $23(54 \%)$ & 0.463 \\
\hline
\end{tabular}

Data are reported as mean \pm SD, median (interquartile range), or number (\%). ASA: American Society of Anesthesiologists, RBC: red blood cells, APACHE: Acute Physiology and Chronic Health Evaluation, ICU: intensive care unit.

ly higher mortality rates at 90 days $(33.6 \%$ vs. $4.7 \%, \mathrm{P}<0.001)$ and one year $(40.3 \%$ vs. $8.6 \%, \mathrm{P}<0.001)$ (Fig. 2 ).

Table 2 shows that stage 2 and higher pressure ulcers were primarily located on the coccyx (79.1\%), heel (9.3\%), and occiput (7.0\%). The univariate analysis revealed that pressure ulcer development was significantly associated with preoperative hemoglobin levels, albumin levels, lactate levels, intraoperative blood loss and number of $p \mathrm{RBC}$ units, postoperative APACHE score, Braden scale score, postoperative ventilator care, and physical restraint (Table 3). After considering multicollinearity between multiple variables, intraoperative preoperative albumin, and lactate levels, the intraoperative number of $p \mathrm{RBC}$ units, postoperative ventilator care, and Braden scale score were entered into the multiple logistic regression model. Pressure ulcer development was independently predicted by lower preoperative albumin levels (odds ratio [OR]: 0.2, 95\% CI: 0.05-0.82; P = 
0.025) and higher lactate levels (OR: 1.7, 95\% CI: 1.07-2.71; P = 0.026). The number of $p \mathrm{RBC}$ units, postoperative Braden scale score, and postoperative ventilator care were not significantly associated with the risk of pressure ulcer development in the multivariate analysis (all $\mathrm{P}>0.05$ ).

Based on these findings, we constructed a clinical model for predicting postoperative pressure ulcer development. The model also included the number of $p \mathrm{RBC}$ units, postoperative ventilator care, and Braden scale score, because they are known risk factors for pressure ulcer development. The model's predictive power was tested using an ROC curve, which had an AUC of 0.88 (95\% CI: 0.79-0.97; P < 0.001). Fig. 3 shows the nomogram, and the validation set was used to compare the nomogram-predicted pressure ulcer rate to the observed rate. The nomogram accurately predicted postoperative pressure ulcer development (Fig. 4).

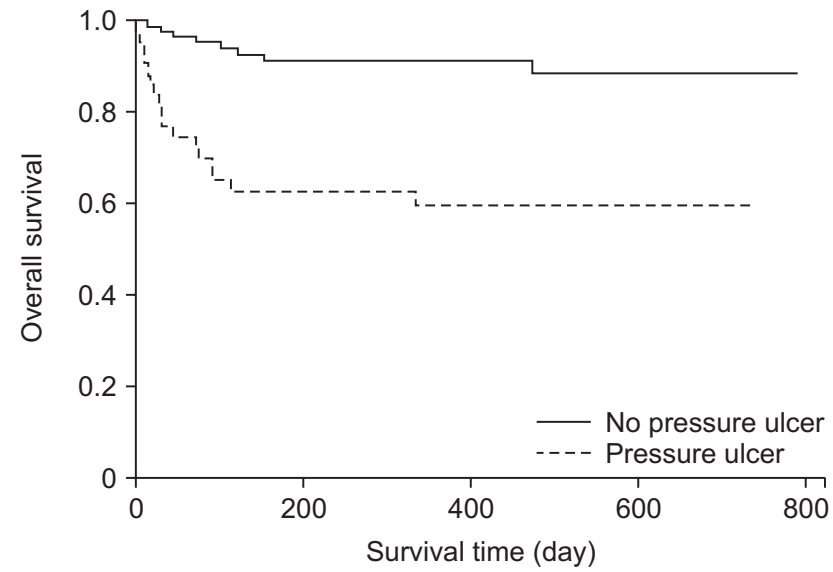

Fig. 2. Kaplan-Meier survival curves of no pressure ulcer group (straight line, $n=86$ ) and pressure ulcer group (dotted line, $n=43$ ) postoperative pressure ulcer. Patient in the pressure ulcer group had a significantly lower survival rate (90-day predictive mortality rate, $33.6 \%$ vs. $4.7 \%$; 1 -year predictive mortality rate, $40.3 \%$ vs. $8.6 \%$; both $\mathrm{P}<0.001$ ).

\section{Discussion}

The present study aimed to identify perioperative factors that predicted postoperative pressure ulcers among surgical patients and revealed that preoperative low albumin levels and high lactate levels were independent risk factors. Previous studies have reported broad variability in the rate of pressure ulcer development in the surgical setting $(2.8-12 \%)$ [10,11], and the present study revealed that $10.2 \%$ of patients had stage 2 or higher postoperative pressure ulcers. After we excluded patients with preoperative pressure ulcers, we found that the incidence of postoperative pressure ulcers was $3.7 \%$ (Fig. 1).

There is an increasing amount of literature regarding the risk factors for postoperative pressure ulcers $[4,12,13]$. For example, patients who undergo surgical procedures are immobile for prolonged periods, are unable to change positions, and have a higher risk of pressure ulcers, compared to mobile patients [14]. Furthermore, patients under general anesthesia cannot sense numbness or pain and are unable to change position to relieve focal pressure. Although patients are positioned intraoperatively to reduce pressure, pressure ulcers remain fairly common among patients who require postoperative critical care. One literature review has indicated that the proposed mechanism for pressure ulcer development is multi-factorial [15]. For example, mechanical loading may be sufficient to cause occlusion of capillaries, which results in ischemia and cell death [15]. In addition, intrin-

Table 2. Pressure Ulcer Sites

\begin{tabular}{lc}
\hline & $\mathrm{n}(\%)$ \\
\hline Occiput & $3(7)$ \\
Coccyx & $34(79)$ \\
Heel & $4(9)$ \\
Back & $2(5)$ \\
Total & $43(100)$
\end{tabular}

Table 3. Power of Selected Variables for Predicting Pressure Ulcer Formation

\begin{tabular}{|c|c|c|c|c|}
\hline & \multicolumn{2}{|c|}{ Univariate analysis } & \multicolumn{2}{|c|}{ Multivariate analysis } \\
\hline & Odds ratio $(95 \% \mathrm{CI})$ & $\mathrm{P}$ value & Odds ratio $(95 \% \mathrm{CI})$ & $P$ value \\
\hline Preoperative hemoglobin (g/dl) & $0.80(0.66-0.97)$ & 0.020 & & \\
\hline Preoperative albumin (g/dl) & $0.29(0.16-0.53)$ & $<0.001$ & $0.21(0.05-0.82)$ & 0.025 \\
\hline Preoperative lactate $(\mathrm{mmol} / \mathrm{L})$ & $1.73(1.15-2.60)$ & 0.009 & $1.70(1.07-2.71)$ & 0.026 \\
\hline Blood loss (L) & $1.00(1.00-1.00)$ & 0.048 & & \\
\hline Packed RBC transfusion (units) & $1.07(1.01-1.15)$ & 0.028 & $0.99(1.92-1.06)$ & 0.772 \\
\hline APACHE II score & $1.08(1.03-1.15)$ & 0.005 & & \\
\hline Braden scale & $0.75(0.64-0.88)$ & $<0.001$ & $0.88(0.64-1.21)$ & 0.421 \\
\hline Ventilator care & $6.92(2.49-19.28)$ & $<0.001$ & $0.14(0.10-1.92)$ & 0.140 \\
\hline Restraint & $8.88(2.92-27.03)$ & $<0.001$ & & \\
\hline
\end{tabular}

RBC: red blood cells, APACHE: Acute Physiology and Chronic Health Evaluation, Ventilator care: postoperative ventilator care, Restraint: physical restraint used in intensive care unit. 


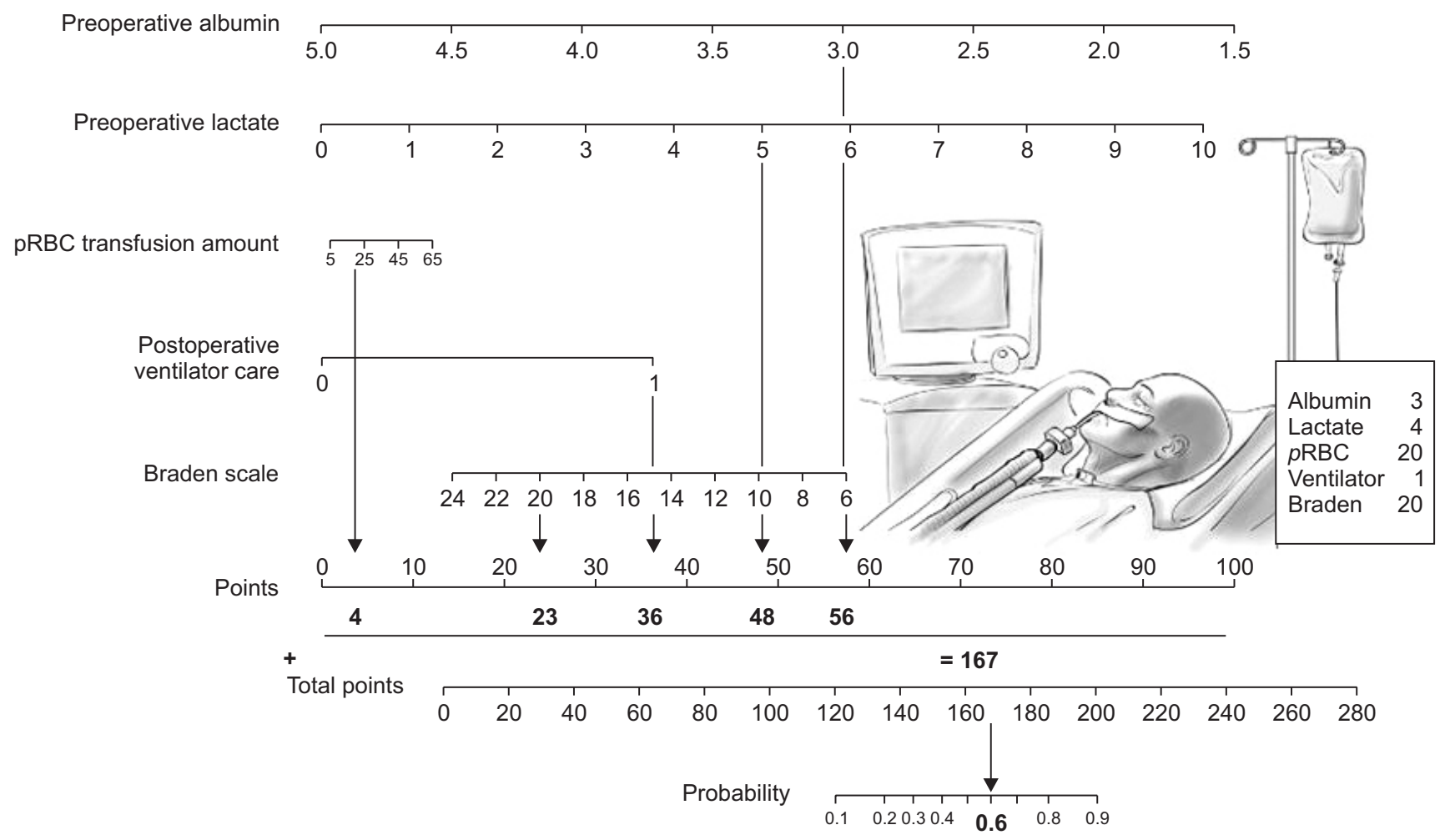

Fig. 3. Nomogram to predict the probability of postoperative pressure ulcer (Top). Points are obtained according to the prognostic contribution of parameters (Bottom). Points are translated to the probability of requiring postoperative pressure ulcer. Predictor points are found on the uppermost point scale that corresponds to each individual variable. The reader then manually sums the points, and the predicted values can be read at the bottom of the nomogram. The total projected on the bottom scale indicates the probability of postoperative pressure ulcer. For example, a patient is admitted to the ICU after major surgery. Preoperative laboratory data shows that albumin is $3.0 \mathrm{~g} / \mathrm{dl}$ and lactate is $4.0 \mathrm{mmol} / \mathrm{L}$. During the surgery, $20 \mathrm{units}$ of packed RBC units are transfused. Postoperatively, he is transferred to ICU and needed a mechanical ventilator support for a while. At that time, Braden scale assessed by a nurse is 20 . In this case, the incidence of postoperative pressure ulcer is expected as much as $60 \%$ by using this nomogram. Therefore, clinician and nursing practioners should be more concerned to prevent pressure ulcer for this patient.

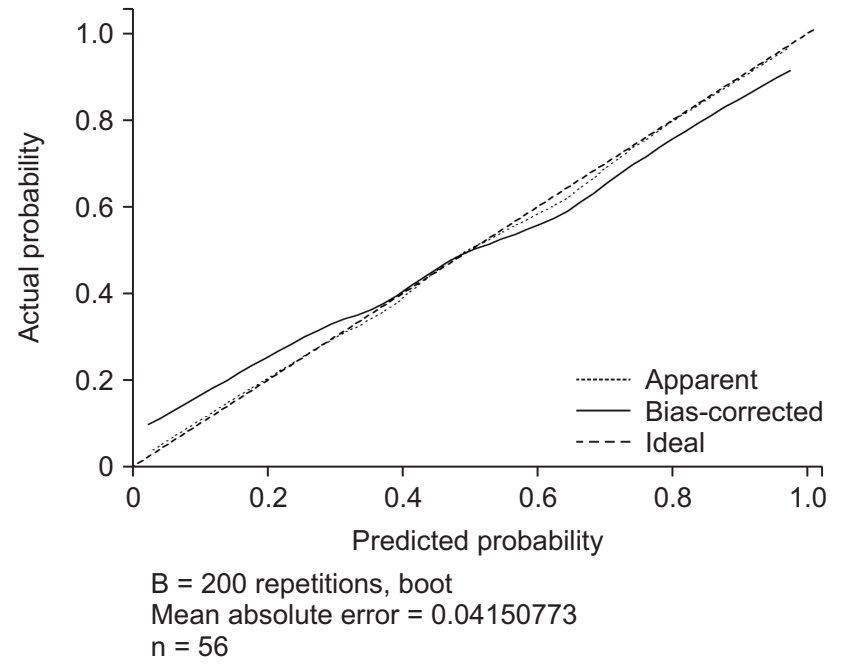

Fig. 4. Internal validation of the nomogram to predict postoperative pressure ulcer. Predictive accuracy of the model (nomogram): the frequencies of predicted and actual incidence of postoperative pressure ulcer are plotted as observations. Logistic calibration for the training set: calibration plot $\mathrm{P}=1 ; \mathrm{E}$, difference in predicted and calibrated probabilities between calibration and AUC; $\mathrm{E}$ average $=3.02 \%$. sic patient factors and extrinsic environmental factors have been implicated in pressure ulcer development. The known intrinsic factors include advanced age, comorbidities, poor nutritional status, abnormally low or high BMI, poor circulation, diabetes, and low hematocrit levels. The known extrinsic risk factors include type of anesthesia, duration of surgery, hypothermia, applied moisture, bed type, surgical position, type of surgery, and use of on-pump cardiac surgical procedures $[4,10,11,16,17]$. Thus, the present study used a matched case-control design to exclude unmodifiable extrinsic factors and minimize the effects of confounding factors, and we found that the cases and controls were accurately matched for age ( \pm 5 years), sex, surgical procedure, and comorbidities.

The univariate analysis revealed that postoperative pressure ulcer development was significantly associated with preoperative hemoglobin levels, albumin levels, lactate levels, intraoperative blood loss, $p$ RBC units, postoperative APACHE II score, Braden scale score, postoperative ventilator care, and patient restraint. However, in the multivariate analysis, only baseline lactate and albumin levels were significantly associated with the develop- 
ment of pressure ulcers. In previous studies, van Stijn et al. [18] found that preoperative albumin levels were associated with postoperative outcomes among elderly patients, and Mistrik et al. [19] found that skin blood flow (measured using a laser Doppler line scanner) was significantly associated with serum albumin levels among patients on chronic hemodialysis. Given that circulating albumin levels are assumed to reflect the rate of synthesis, plasma albumin levels are also widely used as an indicator of nutritional status [20,21]. Protein-energy malnutrition is also recognized as a major risk factor for pressure ulcers [22], and the National Pressure Ulcer Advisory Panel and Japanese Society for Parenteral and Enteral Nutrition recommend supplementary high-protein mixed oral nutrition and tube feeding for individuals with a high risk of developing pressure ulcers $[7,23]$. Based on these results, preoperative low albumin levels might indicate poor nutritional status, which might explain its significant positive association with the development of pressure ulcers.

One unanticipated finding of our study was the association of high lactate levels with the development of pressure ulcers ( $P$ $=0.001$ ). Lactate is produced as an end-product of anaerobic metabolism, and normal physiological metabolism does not favor lactate formation, as this pathway only accounts for $10 \%$ of total pyruvate metabolism [24]. Previous studies have confirmed that tissue hypoxia, which is characterized as supply-dependent oxygen consumption can cause elevated levels of lactate $[25,26]$, which are nearly always pathological in cases with prolonged elevation [27,28]. The association between baseline lactate levels and postoperative pressure ulcers is interesting because previous studies have not considered this biomarker, although elevated lactate levels are known to be associated with tissue hypo-perfusion and increased rates of morbidity and mortality [27-29]. Thus, lactate may be a useful biomarker for predicting pressure ulcer development.

Hospital and ICU stays were also associated with pressure ulcer development in the present study and could be both causes and effects of pressure ulcer development. This is because the risk of developing a pressure ulcer theoretically increases during prolonged hospitalization. However, the association may also reflect the patient's poor general condition and subsequent need for a prolonged recovery period (Table 1).

During the immediate postoperative period, grade 1 pressure ulcers are frequently reported at pressure sites based on the patient's intraoperative position. However, the present study only included pressure ulcers of grade 2 or higher, and most of the pressure ulcer sites were on bony prominent areas that contact the table while the patients are in the supine position. Seven patients who developed pressure ulcers underwent surgery in the lateral position, although no pressure ulcers occurred on the pressure bearing areas for this position. Similar results have been observed in an earlier study, as Lumbley et al. [30] reported that the most common pressure ulcer locations were the sacral/coccygeal and buttock regions, which were not correlated with the surgical posture. Although studies have evaluated the pressure interface that is associated with each posture, no studies have evaluated the risk of pressure ulcers based on the patient's surgical position.

Based on our newly identified risk factors and the known risk factors ( $p \mathrm{RBC}$ units, postoperative ventilator use, and Braden scale score), we developed a simple nomogram to predict postoperative pressure ulcers among surgical patients. The model was able to identify patients who developed pressure ulcers and provided an AUC of 0.88 (95\% CI: 0.79-0.97; P < 0.001). These results indicate that a patient's risk of pressure ulcer development can be accurately predicted by combining their preoperative and intraoperative data. As our nomogram could identify patients who were at risk for pressure ulcer development, it may be useful for guiding more appropriate positioning and the use of a pressure-reducing surface for these patients.

The most important limitation of this study is its casecontrol design, which is associated with known risks of recall bias and exposure misclassification. In addition, we focused on modifiable factors that were associated with the risk of pressure ulcers, although numerous unmodifiable factors have already been identified (e.g., the surgical procedure). Furthermore, the limited sample size is also associated with known risks of bias. Moreover, our study requires external validation to confirm the accuracy and reproducibility of our findings. Therefore, future research is needed to test our model, and to investigate the role of preoperative nutritional support in preventing pressure ulcer development.

In conclusion, we evaluated perioperative factors that may predict pressure ulcer development after surgery and found that preoperative low albumin levels and high lactate levels were independent risk factors. Using these newly identified risk factors, as well as previously established risk factors, we developed a simple clinical nomogram for predicting postoperative pressure ulcer development among surgical patients. This nomogram may allow clinicians to plan the patient's postoperative ICU care and develop strategies to prevent pressure ulcer development, which may reduce the associated rates of morbidity and mortality.

\section{Acknowledgments}

The authors thank Dong-Su Jang, MFA (Medical Illustrator, Medical Research Support Section, Yonsei University College of Medicine, Seoul, Korea) for his help with the illustrations. 


\section{ORCID}

Jeong Min Kim, https://orcid.org/0000-0025-0468-8012

Hyunjeong Lee, https://orcid.org/0000-0002-3980-9697

Taehoon Ha, https://orcid.org/0000-0001-9035-8003

Sungwon Na, https://orcid.org/0000-0002-1170-8042

\section{References}

1. Kandilov AM, Coomer NM, Dalton K. The impact of hospital-acquired conditions on Medicare program payments. Medicare Medicaid Res Rev 2014; 4.

2. Nilsson UG. Intraoperative positioning of patients under general anesthesia and the risk of postoperative pain and pressure ulcers. J Perianesth Nurs 2013; 28: 137-43.

3. He W, Liu P, Chen HL. The Braden Scale cannot be used alone for assessing pressure ulcer risk in surgical patients: a meta-analysis. Ostomy Wound Manage 2012; 58: 34-40.

4. O’Brien DD, Shanks AM, Talsma A, Brenner PS, Ramachandran SK. Intraoperative risk factors associated with postoperative pressure ulcers in critically ill patients: a retrospective observational study. Crit Care Med 2014; 42: 40-7.

5. González-Ruiz JM, Sebastián-Viana T, Losa-Iglesias ME, Lema-Lorenzo I, Crespo FJ, Martín-Merino G, et al. Braden Scale and Norton Scale modified by INSALUD in an acute care hospital: validity and cutoff point. Adv Skin Wound Care 2014; 27: 506-11.

6. Hyun S, Vermillion B, Newton C, Fall M, Li X, Kaewprag P, et al. Predictive validity of the Braden scale for patients in intensive care units. Am J Crit Care 2013; 22: 514-20.

7. Edsberg LE, Black JM, Goldberg M, McNichol L, Moore L, Sieggreen M. Revised national pressure ulcer advisory panel pressure injury staging system: revised pressure injury staging system. J Wound Ostomy Continence Nurs 2016; 43: 585-97.

8. Berman AJ, Snyder S, Kozier BJ, Erb G. Fundamentals of nursing: concepts, process, and practice. 8th ed. New Jersey, Pearson Education. 2008, pp 905-7.

9. Graesslin O, Abdulkarim BS, Coutant C, Huguet F, Gabos Z, Hsu L, et al. Nomogram to predict subsequent brain metastasis in patients with metastatic breast cancer. J Clin Oncol 2010; 28: 2032-7.

10. Hayes RM, Spear ME, Lee SI, Krauser Lupear BE, Benoit RA, Valerio R, et al. Relationship between time in the operating room and incident pressure ulcers: a matched case-control study. Am J Med Qual 2015; 30: 591-7.

11. Tschannen D, Bates O, Talsma A, Guo Y. Patient-specific and surgical characteristics in the development of pressure ulcers. Am J Crit Care 2012; $21: 116-25$.

12. Hayes RM, Spear ME, Lee SI, Krauser Lupear BE, Benoit RA, Valerio R, et al. Relationship between time in the operating room and incident pressure ulcers: a matched case-control study. Am J Med Qual 2015; 30: 591-7.

13. Engels D, Austin M, McNichol L, Fencl J, Gupta S, Kazi H. Pressure ulcers: factors contributing to their development in the OR. AORN J 2016; 103: 271-81.

14. Lindgren M, Unosson M, Fredrikson M, Ek AC. Immobility--a major risk factor for development of pressure ulcers among adult hospitalized patients: a prospective study. Scand J Caring Sci 2004; 18: 57-64.

15. Coleman S, Nixon J, Keen J, Wilson L, McGinnis E, Dealey C, et al. A new pressure ulcer conceptual framework. J Adv Nurs 2014; 70: 222234.

16. Roberts S, Chaboyer W, Leveritt M, Banks M, Desbrow B. Nutritional intakes of patients at risk of pressure ulcers in the clinical setting. Nutrition 2014; 30: 841-6.

17. Liu P, He W, Chen HL. Diabetes mellitus as a risk factor for surgery-related pressure ulcers: a meta-analysis. J Wound Ostomy Continence Nurs 2012; 39: 495-9.

18. van Stijn MF, Korkic-Halilovic I, Bakker MS, van der Ploeg T, van Leeuwen PA, Houdijk AP. Preoperative nutrition status and postoperative outcome in elderly general surgery patients: a systematic review. JPEN J Parenter Enteral Nutr 2013; 37: 37-43.

19. Mistrík E, Dusilová-Sulková S, Bláha V, Sobotka L. Plasma albumin levels correlate with decreased microcirculation and the development of skin defects in hemodialyzed patients. Nutrition 2010; 26: 880-5.

20. Yatabe MS, Taguchi F, Ishida I, Sato A, Kameda T, Ueno S, et al. Mini nutritional assessment as a useful method of predicting the development of pressure ulcers in elderly inpatients. J Am Geriatr Soc 2013; 61: 1698-704.

21. Hülshoff A, Schricker T, Elgendy H, Hatzakorzian R, Lattermann R. Albumin synthesis in surgical patients. Nutrition 2013; 29 : $703-7$.

22. Litchford MD, Dorner B, Posthauer ME. Malnutrition as a precursor of pressure ulcers. Adv Wound Care (New Rochelle) 2014; 3: 54-63.

23. Revision TJSPUG: Japanese Society of Pressure Ulcers: guidelines for the prevention and management of pressure ulcer. 3rd ed. Tokyo, shorinsha. 2014, pp 12-90.

24. Vernon C, Letourneau JL. Lactic acidosis: recognition, kinetics, and associated prognosis. Crit Care Clin 2010; 26: 255-83. 
25. Cain SM. Oxygen delivery and uptake in dogs during anemic and hypoxic hypoxia. J Appl Physiol Respir Environ Exerc Physiol 1977; 42: 228-34.

26. Friedman G, De Backer D, Shahla M, Vincent JL. Oxygen supply dependency can characterize septic shock. Intensive Care Med 1998; 24: 118-23.

27. Okorie ON, Dellinger P. Lactate: biomarker and potential therapeutic target. Crit Care Clin 2011; 27: 299-326.

28. Kjelland CB, Djogovic D. The role of serum lactate in the acute care setting. J Intensive Care Med 2010; 25: 286-300.

29. Haidl F, Brabrand M, Henriksen DP, Lassen AT. Lactate is associated with increased 10-day mortality in acute medical patients: a hospitalbased cohort study. Eur J Emerg Med 2015; 22: 282-4.

30. Lumbley JL, Ali SA, Tchokouani LS. Retrospective review of predisposing factors for intraoperative pressure ulcer development. J Clin Anesth 2014; 26: 368-74. 